\title{
Correlation between morphology of optic disc determined by Heidelberg Retina Tomograph II and visual function in eyes with open-angle glaucoma
}

\author{
This article was published in the following Dove Press journal: \\ Clinical Ophthalmology \\ 10 July 2010 \\ Number of times this article has been viewed
}

\author{
Kazuko Omodaka \\ Toru Nakazawa \\ Takaaki Otomo \\ Masahiko Nakamura \\ Nobuo Fuse \\ Kohji Nishida \\ Department of Ophthalmology, \\ Tohoku University Graduate School \\ of Medicine, I-I Seiryo-machi, \\ Aoba-ku, Sendai, Miyagi 980-8574, \\ Japan
}

\begin{abstract}
Purpose: To investigate the correlation between the morphology of the optic disc and the visual function of eyes with open-angle glaucoma (OAG).

Patients and Methods: One hundred and three eyes of 76 patients with OAG were studied. The baseline optic disc morphology was used to classify the eyes into four types: focal ischemic type (FI), myopic glaucomatous type (MY), senile sclerotic type (SS), and generalized enlargement type (GE). The morphological parameters of the disc were determined by the Heidelberg Retina Tomograph II (HRT-II) and the visual function by the mean deviation (MD) of the Humphrey field analyzer.
\end{abstract}

Results: Fourteen eyes were classified as the FI type; 52 as the MY type; 17 as the SS type; and 20 as the GE type. The highest correlation coefficients of HRT-II parameters to the MDs was the cup/disc area ratio $(r=-0.27)$ for all groups, the vertical cup/disc ratio $(r=-0.42)$ in the MY group, the maximum cup depth $(r=0.49)$ in the SS group, and the cup area $(r=-0.70)$ in the GE group. However, none of the parameters was correlated in the FI group.

Conclusions: The correlation between the HRT-II parameters and MDs was different for the four disc types. These findings suggest that a classification of optic disc morphology had a benefit for interpreting the measured values of HRT-II.

Keywords: open angle glaucoma, optic disc morphology, classification, visual function

\section{Introduction}

Glaucoma affects over 70 million people worldwide, and is the second most common cause of blindness. ${ }^{1,2}$ Glaucoma is characterized by optic nerve fiber atrophy that causes a decrease in vision. ${ }^{3}$ Although an elevated intraocular pressure (IOP) is generally recognized as a major risk factor for glaucoma, ${ }^{4}$ it is well known that glaucoma is a multifactorial disease. For the majority of eyes with open-angle glaucoma (OAG), lowering the IOP is currently the only standard treatment to prevent the progression of the disease. However, some patients with a significant IOP reduction ${ }^{5}$ or even those with normal IOPs ${ }^{6}$ still show a progression of the glaucomatous changes even to blindness.

It is difficult to detect the early changes of glaucoma with standard automated perimetry (SAP) because nerve fiber degeneration and loss of visual fields do not progress in parallel in the early stage of glaucoma. ${ }^{7}$ A number of studies have shown that the visual field abnormalities are detected only after $20 \%-50 \%$ of the retinal ganglion cells have been lost..$^{8-10}$ The early changes in patients with OAG are those in
Correspondence:T Nakazawa

Department of Ophthalmology, Tohoku University Graduate School of Medicine,

I-I Seiryo-machi, Aoba-ku, Sendai,

Miyagi 980-8574, Japan

Tel +8I 227177294

Fax $+8 \mid 227177298$

Email ntoru@oph.med.tohoku.ac.jp 
the thickness of the nerve fiber layer and in the morphology of the optic disc. ${ }^{11,12}$ To assess and follow these changes, modern instruments based on laser confocal systems or optical coherence systems are used. ${ }^{13-15}$ Importantly, the findings of these instruments are correlated with the different degrees of the mean deviations (MDs) of the Humphrey field analyzer (HFA). ${ }^{16-18}$ If it is possible to improve the correlation between the objective results of the instruments and the subjective results of SAP, the instruments would be more valuable for the diagnosis and the follow-up of patients with OAG.

Various patterns of optic disc alterations have been recognized in patients with glaucoma. Nicolela and colleagues classified the optic disc appearances into four types: the focal ischemic (FI) disc type; myopic (MY) disc type; senile sclerotic (SS) disc type; and generalized enlargement (GE) disc type. ${ }^{19}$ They found that patients with SS were less likely to have a progression of the optic disc morphology and visual field defects than patients with the other types of discs with similar IOPs. ${ }^{20}$

The Heidelberg Retinal Tomograph-II (HRT-II) is a confocal scanning laser imaging instrument that measures the 3-dimensional topography of the optic nerve head. Different features of the optic disc morphology are calculated from the measurements and used to characterize the morphology of the optic disc.

Because of the reliability of the HRT-II, ${ }^{21,22}$ we believed that objective determinations of the morphology of the optic disc obtained by HRT-II can be used to classify the optic discs into different morphological types. We hypothesized that the correlation coefficients between the optic disc parameters and the MD of the HFA will be higher when the parameters of the different types of optic disc morphology are individually assessed. To accomplish this, the optic disc morphology was classified into the same four types presented by Nicolela and colleagues ${ }^{19}$ and the optic disc parameters for each of these types was correlated with the MDs obtained from the HFA of each patient.

\section{Patients and methods Inclusion criteria}

This was a retrospective study of the clinical records of patients with glaucoma. Patients were included if they had the following: diagnosed with primary open angle glaucoma (POAG: baseline IOP $>22 \mathrm{mmHg}$ ) or normal tension glaucoma (NTG: baseline IOP $\leq 21 \mathrm{mmHg}$ ); spherical equivalent myopia greater than -8 diopters; abnormal glaucomatous visual field according to Anderson-Pattela classification ${ }^{23}$ or best-corrected visual acuity $>20 / 30$; and
MD of HFA $>-12 \mathrm{~dB}$. Patients were excluded if: ocular diseases except for OAG were present; systemic diseases affecting the visual fields were present; had undergone intraocular surgery; and cataract progression during the follow-up period. The baseline clinical parameters including age, gender, and refractive error for each patient were recorded. The IOP was measured by applanation tonometry and the baseline IOP was the average of at least three measurements without any medications.

\section{Classification of disc appearances}

The optic discs were divided into four types following the classification of Nicolela and colleagues ${ }^{19,20}$ (Figure 1); focal ischemic (FI) disc type; myopic (MY) disc type; senile sclerotic (SS) disc type; and generalized enlargement (GE) disc type. The FI type of optic disc had a focal loss of nerve fibers on the neuronal rim and the other areas were normal. In the MY type, there was temporal parapapillary atrophy with temporal cupping with slight tilting of the disc. In the SS type, there was an atrophic halo (chorioretinal atrophy) around the optic disc with shallow cupping. In the GE type, a large and deep cupping, especially nasally, was present. Two glaucoma specialists performed the classification of the disc types independently in good quality optic disc photographs, and if their classification did not agree and the optic disc was difficult to classify, the case was excluded (24.9\%).

\section{Visual field analyses}

The MD values were obtained by the Swedish interactive threshold algorithm (SITA)-standard strategy of the 30-2 program of HFA (Carl Zeiss Meditec, Dublin, CA, USA). The MDs of only the reliable visual fields ( $<20 \%$ fixation errors, $<33 \%$ false-positives, and $<33 \%$ false-negatives) and measured within three months of HRT-II examination were used in the analyses.

\section{Optic disc measurements with HRT-II}

HRT-II uses a diode laser (670-nm wavelength) that sequentially scans the retinal surface in both the horizontal and vertical directions in different focal planes. The optic disc contour line was drawn manually at the inner edge of the sclera ring by one experienced glaucoma specialist. The patient's pupils were dilated before the examination because this has been reported to improve the quality of the HRT-II images. ${ }^{24}$ Fourteen HRT parameters: the disc area; rim area; cup volume; cup area; cup/disc area ratio; rim volume; mean cup depth; vertical cup/disc area ratio; cup shape measure; maximum cup depth; reference height; height variation contour; retinal 

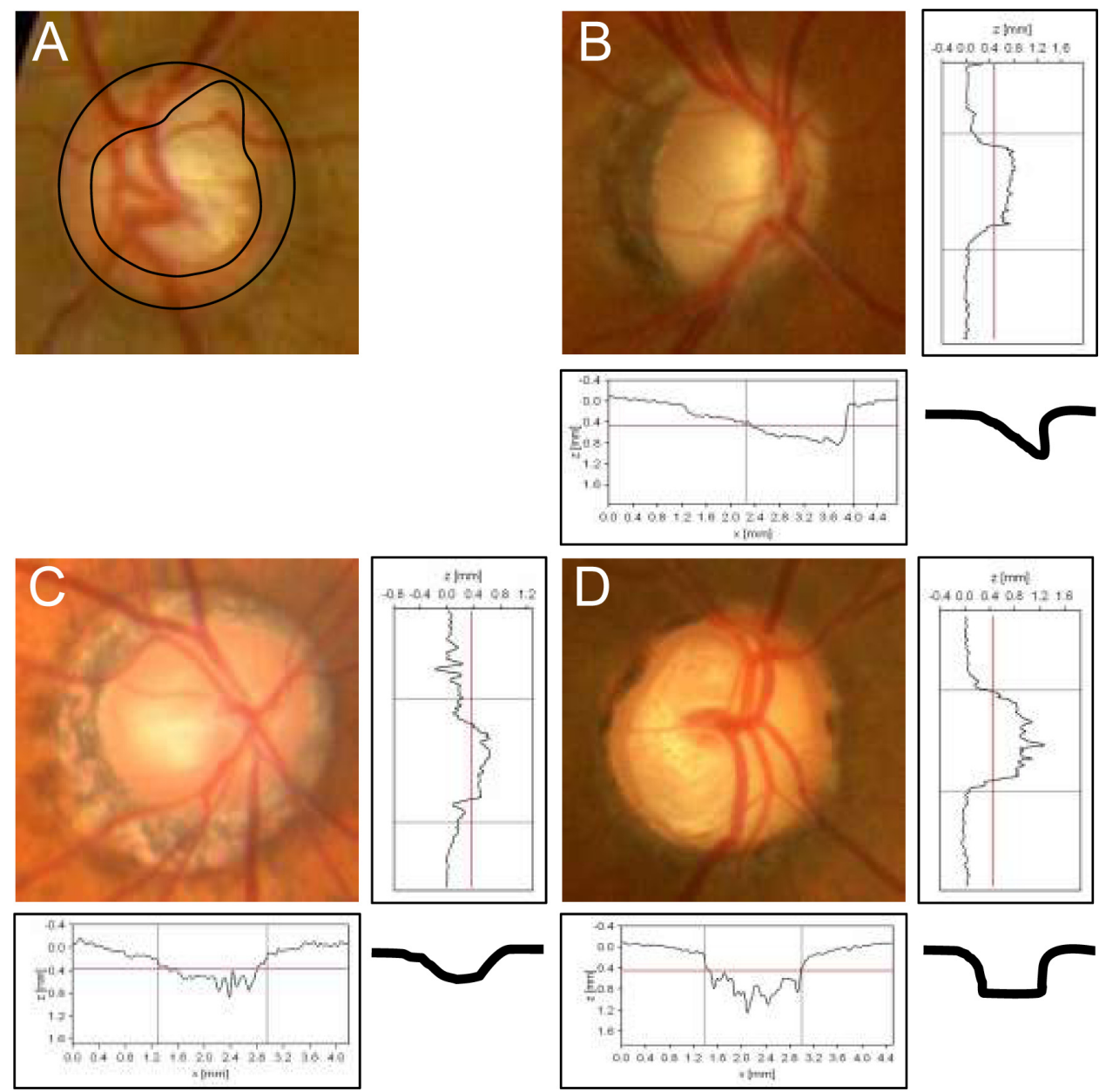

Figure I Representative photographs and schema of the patients with different optic disc types.

Notes: Fl, a focal loss of neuronal rim and the other area of neuronal rim is normal. MY, typical parapapillary atrophy and a shape of temporal cupping with slight tilting. SS, halo (chorioretinal atrophy) surrounding the optic disc with shallow cupping. GE, a large and deep clear cupping, especially nasal cupping.

Abbreviations: $\mathrm{Fl}$, focal ischemia; MY, myopic glaucoma disc; SS, senile sclerosis; GE, generalized enlargement.

nerve fiber layer (RNFL) cross sectional area; and mean RNFL thickness, were determined by the HRT-II software. In addition, the Frederick S Mikelberg (FSM) discriminate function ${ }^{25}$ was calculated by the HRT-II software (version 1.7). If the standard deviation of the mean topographic image was more than $40 \mu \mathrm{m}$, the data were excluded.

\section{Statistical analyses}

Analysis of variance for continuous data (ANOVA) followed by a Scheffe post hoc test was used to determine the significance of the differences in the four groups. Spearman's correlation analyses were performed to determine the correlations between the optic disc parameters obtained by the HRT-II and the MDs obtained by HFA. The significance level was set at $P<0.05$. These analyses were performed with a JMP software (version 8.0.1, SAS Institute Japan, Tokyo).

\section{Results}

Of the 103 eyes of 76 patients: 14 (13\%) optic discs were classified as the FI type; 52 (50\%) as the MY type; 17 (17\%) as the SS type; and 20 (20\%) as the GE type (Figure 1). The MY group was significantly younger (48.4 \pm 13.4 years), and the SS group was significantly older $(67.0 \pm 6.7$ years $)$ than the other groups (Table 1). The refractive error in the MY group was most myopic $(-4.8 \pm 2.7 \mathrm{D})$ among the four groups. The MDs of the HFA and the baseline IOPs were not significantly different for the four discs types (Table 1).

In patients with the FI optic disc type, there were no HRT-II parameters that were significantly different from that of the other types (Table 2). In patients with the MY disc type, the disc area was significantly smaller $(1.92 \pm 0.50)$ than that in the other disc types, and the reference height, retinal nerve fiber layer (RNFL) cross sectional area, and mean RNFL thickness were also significantly different from that of the 
Table I Demographic data of patients with different disc types

\begin{tabular}{llllll}
\hline & FI $(\mathbf{n}=\mathbf{I 4})$ & MY $(\mathbf{n}=\mathbf{5 2})$ & SS $(\mathbf{n}=1 \mathbf{7})$ & GE $(\mathbf{n}=\mathbf{2 0})$ & Total $(\mathbf{n}=\mathbf{I 0 3})$ \\
\hline Sex & & & & & \\
$\quad$ Male, $\mathrm{n}(\%)$ & $\mathrm{I}(7 \%)$ & $24(46 \%)$ & $5(71 \%)$ & $12(60 \%)$ & $42(48 \%)$ \\
$\quad$ Female, $\mathrm{n}(\%)$ & $13(93 \%)$ & $28(54 \%)$ & $12(29 \%)$ & $8(40 \%)$ & $61(52 \%)$ \\
Age, yrs (mean \pm SD) & $60.7 \pm 11.7$ & $48.4 \pm 13.4 *$ & $67.0 \pm 6.7^{*}$ & $52.5 \pm 14.6$ & $54.3 \pm 14.6$ \\
Spherical equivalent (D) & $-0.20 \pm 2.1$ & $-4.8 \pm 2.7^{*}$ & $-1.7 \pm 2.9$ & $-1.2 \pm 2.6$ & $-2.9 \pm 3.2$ \\
MD (dB) & $-3.3 \pm 2.5$ & $-4.6 \pm 3.3$ & $-4.7 \pm 2.7$ & $-3.9 \pm 4.1$ & $-4.3 \pm 3.2$ \\
IOP (mmHg) & $16.5 \pm 2.8$ & $17.2 \pm 5.9$ & $17.2 \pm 5.9$ & $16.9 \pm 7.2$ & $16.9 \pm 5.6$ \\
\hline
\end{tabular}

Notes: $* P<0.05$ (ANOVA).

Abbreviations: SD, standard deviation; FI, focal ischemia; MY, myopic glaucoma disc; SS, senile sclerosis; GE, generalized enlargement; MD, mean deviation of Humphrey fields analyzer; IOP, intraocular pressure; ANOVA, analysis of variance.

other optic disc types. In the patients with the SS disc type, the mean and maximum cup depth was shallower than that in the other disc types. In the patients with the GE type of optic disc: the disc area; rim area; cup volume; cup area; cup/disc area ratio; rim volume; mean cup depth; vertical cup/disc ratio; and cup shape measure, were all significantly different from that in the other optic disc types (Table 2).

There was a weak but significant correlation ( $\mathrm{r}=0.2$ to 0.3 ) between the disc parameters and the MDs in all patients studied (Table 3). When all the disc types were combined, the rim area ( $\mathrm{r}=0.2397, P=0.0038)$, cup/disc area ratio $(\mathrm{r}=-0.2714$, $P=0.0039)$, vertical cup/disc ratio $(\mathrm{r}=-0.240, P=0.0077)$, and cup shape $(\mathrm{r}=-0.2653, P=0.0206)$ were significantly correlated to the MD. The cup/disc area ratio had the highest correlation to the MDs. The FSM discriminate function was significantly correlated to the MDs $(r=0.3135, P=0.001)$.
The coefficients of correlation between the parameters of HRT-II and MDs were different for the different optic discs types. In patients with the FI type, there were no parameters of the HRT-II that was correlated to the MDs. In the patients with the MY type: the rim area $(\mathrm{r}=0.2561$, $P=0.0219)$; cup volume $(\mathrm{r}=-0.2758, P=0.0084)$; cup area $(\mathrm{r}=0.3374, P=0.0185)$; cup/disc area ratio $(\mathrm{r}=-0.3735$, $P=0.0021)$; rim volume $(\mathrm{r}=0.2370, P=0.0001)$; vertical cup/disc ratio $(\mathrm{r}=-0.4197, P=0.0001)$; RNFL cross sectional area $(\mathrm{r}=0.2485, P=0.0365)$; mean RNFL thickness $(\mathrm{r}=0.2716, P=0.0343)$; and FSM $(\mathrm{r}=0.3482, P=0.0122)$; were all significantly correlated with the MDs.

In patients with the SS type of optic disc, only the maximum cup depth $(\mathrm{r}=0.4809, P=0.0396)$ was significantly correlated with the MDs. In the patients with the GE type: the rim area $(r=0.5302, P=0.0388)$; cup area

Table 2 Different characters of HRT-Il parameters in the different disc types

\begin{tabular}{|c|c|c|c|c|c|}
\hline & FI $(n=14)$ & $M Y(n=52)$ & SS $(n=17)$ & GE $(n=20)$ & Total $(n=103)$ \\
\hline Disc area & $2.14 \pm 0.53$ & $1.92 \pm 0.50 *$ & $2.39 \pm 0.42$ & $2.70 \pm 0.6 \mathrm{I} *$ & $2.18 \pm 0.59$ \\
\hline Rim area & $1.01 \pm 0.26$ & $\mathrm{I} . \mathrm{II} \pm 0.46$ & $\mathrm{I} .3 \mathrm{I} \pm 0.37$ & $0.99 \pm 0.37 *$ & $\mathrm{I} . \mathrm{II} \pm 0.4 \mathrm{I}$ \\
\hline Cup volume & $0.36 \pm 0.26$ & $0.18 \pm 0.20$ & $0.16 \pm 0.07$ & $0.63 \pm 0.32 *$ & $0.29 \pm 0.28$ \\
\hline Cup area & $1.13 \pm 0.47$ & $0.81 \pm 0.48$ & $1.08 \pm 0.29$ & $1.70 \pm 0.66 *$ & $1.07 \pm 0.59$ \\
\hline Cup/disc area ratio & $0.51 \pm 0.12$ & $0.40 \pm 0.20$ & $0.45 \pm 0.11$ & $0.62 \pm 0.13 *$ & $0.47 \pm 0.18$ \\
\hline Rim volume & $0.23 \pm 0.07$ & $0.32 \pm 0.19$ & $0.24 \pm 0.08$ & $0.20 \pm 0.12 *$ & $0.27 \pm 0.15$ \\
\hline Mean cup depth & $0.33 \pm 0.10$ & $0.29 \pm 0.11$ & $0.20 \pm 0.04 *$ & $0.42 \pm 0.11 *$ & $0.31 \pm 0.12$ \\
\hline Vertical cup/disc ratio & $0.73 \pm 0.11$ & $0.59 \pm 0.22$ & $0.66 \pm 0.20$ & $0.76 \pm 0.09 *$ & $0.66 \pm 0.20$ \\
\hline Cup shape measure & $-0.07 \pm 0.07$ & $-0.10 \pm 0.08$ & $-0.08 \pm 0.06$ & $-0.03 \pm 0.07 *$ & $-0.08 \pm 0.08$ \\
\hline Maximum cup depth & $0.76 \pm 0.19$ & $0.73 \pm 0.23$ & $0.49 \pm 0.12^{*}$ & $0.87 \pm 0.18$ & $0.72 \pm 0.23$ \\
\hline Reference height & $0.39 \pm 0.08$ & $0.50 \pm 0.12 *$ & $0.31 \pm 0.10$ & $0.38 \pm 0.16$ & $0.43 \pm 0.14$ \\
\hline Height variation contour & $0.55 \pm 0.23$ & $0.53 \pm 0.18$ & $0.38 \pm 0.14$ & $0.42 \pm 0.19$ & $0.49 \pm 0.19$ \\
\hline RNFL cross sectional area & $0.83 \pm 0.24$ & $1.34 \pm 0.47 *$ & $0.76 \pm 0.36$ & $1.08 \pm 0.78$ & $1.12 \pm 0.55$ \\
\hline Mean RNFL thickness & $0.16 \pm 0.04$ & $0.28 \pm 0.10 *$ & $0.13 \pm 0.06$ & $0.18 \pm 0.11$ & $0.22 \pm 0.11$ \\
\hline FSM & $-2.77 \pm 2.69$ & $-1.50 \pm 2.68$ & $-1.14 \pm 1.33$ & $-2.91 \pm 2.44$ & $-1.89 \pm 2.53$ \\
\hline
\end{tabular}

Notes: Bold letter and $* P<0.05$ (ANOVA).

Abbreviations: $\mathrm{Fl}$, focal ischemia; MY, myopic glaucoma disc; SS, senile sclerosis; GE, generalized enlargement; MD, mean deviation of Humphrey fields analyzer; IOP, intraocular pressure; ANOVA, analysis of variance. 
(r $=-0.6959, P=0.008) ; \mathrm{cup} / \mathrm{disc}$ area ratio $(\mathrm{r}=-0.6588$, $P=0.0011)$; cup shape $(\mathrm{r}=-0.6614, P=0.0001)$; and FSM ( $\mathrm{r}=0.767, P=0.0002)$; were significantly correlated with the MDs. In the patients with the GE type, the correlation of the parameters of HRT-II tended to be higher than the other type of disc types (Table 3 ).

\section{Discussion}

We investigated whether there were significant correlations between the disc parameters obtained by HRT-II and the different disc types and also with the MDs of HFA. Thus, the disc area in the patients with the MY type was significantly smaller than that in the other optic disc types, and in the patients with the SS type, the mean and maximum cup depths were significantly shallower than that in the other optic disc types. In the patients with the GE type: the disc area, rim area; cup volume; cup area; cup/disc area ratio; rim volume; mean cup depth; vertical cup/disc ratio; and cup shape measure; were all significantly different from the comparable measures in the other optic disc types. These data suggest that examination with HRT-II helps in the classification of the disc types.

We also found that there were weak but significant correlations between the MDs of HFA and the disc parameters, including: the rim area; cup/disc area ratio; vertical cup/disc ratio; cup shape measure; and FSM in all of the patients with early and middle stage of glaucoma. Our results showed that each optic disc type, except for the FI type, had different HRT-II parameters that were significantly correlated with the MDs, while there were no differences in the baseline of IOPs and MDs among optic disc types. In patients with the MY type, the vertical cup/disc ratio was highly correlated with the MD. In patients with the SS type, the maximum cup depth $(\mathrm{r}=-0.481, P=0.0396)$ and cup area $(\mathrm{r}=-0.696, P=0.0080)$ were highly correlated with the MDs. These data indicate that the HRT-II parameters that have the highest correlation with the MDs are different for each of the four optic disc types. Thus, for a better interpretation of the HRT-II parameters, a classification of the optic disc type should be considered. This would then allow the clinician to know which HRT-II parameter should be followed in glaucoma patients with each disc type.

As reported, when all of the OAG patients are analyzed collectively, the correlations between the parameters of HRT-II and MD were weak. ${ }^{26,27}$ Our results also showed weak correlation coefficients similar to a previous report. ${ }^{27}$ Recently, several research groups have tried to improve the discrimination of normal and early glaucoma using the

Table 3 Correlation between HRT parameters and mean deviation of the patients with different disc types

\begin{tabular}{|c|c|c|c|c|c|c|c|c|c|c|}
\hline & \multicolumn{2}{|c|}{ FI $(n=14)$} & \multicolumn{2}{|c|}{$M Y(n=52)$} & \multicolumn{2}{|c|}{ SS $(n=17)$} & \multicolumn{2}{|c|}{ GE $(n=20)$} & \multicolumn{2}{|c|}{ Total $(n=103)$} \\
\hline & $\mathbf{r}$ & $P$ & $\mathbf{r}$ & $P$ & $\mathbf{r}$ & $P$ & $\mathbf{r}$ & $P$ & $\mathbf{r}$ & $\mathbf{P}$ \\
\hline Disc area & 0.2840 & 0.1933 & -0.0916 & 0.4063 & -0.0141 & 0.8407 & -0.4353 & 0.3607 & -0.0854 & 0.9607 \\
\hline Rim area & 0.1140 & 0.4911 & $0.256 \mathrm{I}$ & 0.0219 & $0.07 \mid 4$ & 0.7504 & 0.5302 & 0.0388 & 0.2397 & 0.0038 \\
\hline Cup volume & 0.4370 & 0.1713 & -0.2758 & 0.0084 & 0.1300 & 0.8699 & -0.5489 & 0.0670 & -0.1397 & 0.3494 \\
\hline Cup area & 0.2598 & 0.2697 & 0.3374 & 0.0185 & -0.1113 & 0.6975 & -0.6959 & 0.0080 & -0.2317 & 0.0898 \\
\hline Cup/disc area ratio & 0.1256 & 0.3617 & -0.3735 & 0.0021 & -0.1191 & 0.4679 & -0.6588 & 0.0011 & -0.2714 & 0.0039 \\
\hline Rim volume & 0.0331 & 0.7081 & 0.2370 & 0.0105 & 0.1095 & 0.7010 & 0.3724 & 0.0540 & 0.1865 & 0.0089 \\
\hline Mean cup depth & 0.3634 & 0.1560 & -0.2306 & 0.0893 & 0.3334 & 0.2083 & -0.2416 & 0.5714 & -0.0600 & 0.9842 \\
\hline $\begin{array}{l}\text { Vertical cup/disc } \\
\text { ratio }\end{array}$ & 0.1987 & 0.4133 & -0.4197 & 0.0001 & -0.0787 & 0.8115 & -0.4392 & 0.0559 & -0.2404 & 0.0077 \\
\hline Cup shape measure & 0.0600 & 0.7194 & -0.2621 & 0.1913 & -0.2891 & 0.3653 & -0.6614 & 0.0001 & -0.2653 & 0.0206 \\
\hline Maximum cup depth & 0.1967 & 0.1415 & -0.1726 & 0.3068 & 0.4809 & 0.0396 & 0.1500 & 0.3959 & 0.0331 & 0.4370 \\
\hline Reference height & -0.3532 & 0.0571 & -0.0200 & 0.8711 & -0.0500 & 0.9330 & -0.2142 & 0.8551 & -0.1157 & 0.4808 \\
\hline $\begin{array}{l}\text { Height variation } \\
\text { contour }\end{array}$ & 0.2901 & 0.5886 & -0.1307 & 0.6942 & 0.0400 & 0.4304 & -0.3874 & 0.0744 & -0.1053 & 0.4315 \\
\hline $\begin{array}{l}\text { RNFL cross } \\
\text { sectional area }\end{array}$ & -0.1174 & 0.7534 & 0.2485 & 0.0365 & 0.3170 & 0.2684 & 0.0346 & 0.1814 & 0.1109 & 0.0329 \\
\hline $\begin{array}{l}\text { Mean RNFL } \\
\text { thickness }\end{array}$ & -0.2483 & 0.2290 & 0.2716 & 0.0343 & 0.3404 & 0.1180 & 0.1542 & 0.2021 & 0.1414 & 0.0636 \\
\hline FSM & -0.2000 & 0.5270 & 0.3482 & 0.0122 & 0.2172 & 0.4562 & 0.7670 & 0.0002 & 0.3135 & 0.0010 \\
\hline
\end{tabular}

Notes: Table showed the correlation coefficient $(r)$ and $P$ value analyzed by Spearman's correlation analysis. $* P<0.05$.

Abbreviations: Fl, focal ischemia; MY, myopic glaucoma disc; SS, senile sclerosis; GE, generalized enlargement; RNFL, retinal nerve fiber layer. 
HRT-II parameters. Thus, Saarela and colleagues showed that a combination of three parameters, viz., the maximum cup depth, the linear cup/disc area ratio, and the horizontal cup/disc area ratio, improved the chances of identifying glaucomatous eyes. ${ }^{17}$ The FSM, ${ }^{25}$ Reinhard OW Burk (RB), ${ }^{28}$ and Bathija formula ${ }^{29}$ were developed to identify glaucomatous eyes from the HRT-II parameters. The FSM was demonstrated to have the highest sensitivity, ${ }^{15}$ and our results showed that the FSM discriminate function was significantly correlated with the MDs of the HFA ( $\mathrm{r}=0.3135, P=0.001)$.

In patients with the GE type, several of the parameters of the optic discs obtained by HRT-II were significantly different from those in the other groups. The patients with GE had a larger disc area, deeper cup depth, larger cup area and volume, and thinner rim area. These data may suggest that the parameters of HRT-II are well reflected by the cup shape of the GE type. Thus, we hypothesized that the classification of optic disc shape would help improve the correlation between the parameter of HRT-II and the MDs, which would then result in an improvement of the diagnosis. Statistical analyses of the four disc types showed that the correlation of the disc parameters and MD were higher in the patients with the GE type optic disc than for the other disc types, and the cup area had the highest correlation coefficient of all the parameters $(r=-0.6959, P=0.008)$. Therefore to follow glaucoma patients with the GE type of optic disc, the optic disc area would be the best parameter to follow to determine whether the glaucomatous changes were progressing.

The optic disc area of the patients with the MY type was the smallest. The average refractive error in the patients with MY was $-4.0 \mathrm{D}$, and this was significantly more myopic than that in the other groups. Fifty percent of the eyes had the MY type of optic disc, and this may be related to the higher prevalence of myopia in the Japanese $\mathrm{e}^{30,31}$ than in western countries. ${ }^{32,33}$ The Tajima Study, a population-based study in Japan, ${ }^{34}$ showed that the disc size was significantly smaller in myopic eyes. This is relevant because the disc size affects the different parameters of HRT-II. ${ }^{35}$ Nakamura and colleagues $^{36}$ found that the mean and maximum cup depth significantly increased with an increase in myopia in normal Japanese subjects although the sample size was small. Thus consistent with our results, the refractive error may affect the parameters of HRT-II.

We found that the vertical cup/disc ratio had the highest correlation coefficient to the MD among all of the parameters of HRT-II. Several research groups have discussed the limitation of the examination of a titled disc, a characteristic of the MY disc type. The inaccuracy in detecting a titled disc may arise from the reference line in the software analysis, and there is a tendency to underestimate the degree of cupping in the patients with the MY type of optic disc. In the current version of the HRT-II software, the reference line was fixed $50 \mu \mathrm{m}$ posterior to the mean contour line height between $350^{\circ}$ and $356^{\circ}$ of the temporal margin of the optic nerve head. Generally in the patients with the MY type, the height between temporal and nasal was significantly different. New methods to determine the reference plane have been published which can improve the ability of the HRT to detect early glaucoma, especially in eyes with a tilted disc. ${ }^{37,38}$ With the standard version of HRT-II analysis software, the vertical cup/disc ratio may be the least influenced parameter in the tilted disc. Thus, it is best to follow the vertical cup/disc ratio in the patients with the MY type of optic disc which is the major phenotype in Asia.

A significantly shallower mean and maximum cup depth was found in patients with the SS type than in the other disc group. The average age of this group was older than the other groups, and they have the best prognosis. ${ }^{20}$ A lower diastolic velocity and a higher resistance index ${ }^{39}$ were shown in patients with this type of disc. In addition, shallower maximum cup depth is associated with worse MD ( $\mathrm{r}=0.4809, P=0.0396$ ) in the patients with the SS type, and this parameter was significantly correlated with the MD in only the SS type. Generally, glaucoma is characterized by deep and undermined cupping of the optic disc, and it is completely the opposite of the shallower cupping associated with the worse visual fields in the patients with the SS type. These data suggest that the mechanism of axonal damage in SS may be different from that in the other disc types.

In the patients with FI, none of the parameters were significantly correlated with the MD. This is reasonable because the damage to the axons on the optic disc is focal and the generalized parameters of HRT-II are difficult to detect. On the other hand, the damaged area of the optic disc and visual fields defect should be closely related in this disc type compared to the other disc type. ${ }^{26,40}$ Thus in the patients with FI, we should use more local parameters than whole disc areas. Moreover, sectional evaluation of the visual fields such as pattern standard deviation (PSD), but not MD, would be more appropriate to compare to the parameters of the HRT-II.

In conclusion, HRT-II is valuable for determining the morphological characteristics of the optic disc, and 
there are low to moderate significant correlations between the parameters of HRT-II and MDs in all patients with glaucoma. However, the association of the parameters of HRT-II and MD of HFA depends on the optic disc appearance, and the correlations are higher if the parameters were analyzed for the different optic disc types. Our findings suggest that the HRT-II measurements would be more useful when they are applied to the different optic disc types.

\section{Acknowledgment}

The authors thank Prof Duco Hamasaki for editing this manuscript.

\section{Disclosure}

The authors report no conflicts of interest relevant to this research.

\section{References}

1. Quigley HA. Number of people with glaucoma worldwide. $\mathrm{Br} J$ Ophthalmol. 1996;80:389-393.

2. Resnikoff S, Pascolini D, Etya'ale D, et al. Global data on visual impairment in the year 2002. Bull World Health Organ. 2004;82:844-851.

3. Weinreb RN, Khaw PT. Primary open-angle glaucoma. Lancet. 2004;363:1711-1720.

4. Kass MA, Heuer DK, Higginbotham EJ, et al. The Ocular Hypertension Treatment Study: a randomized trial determines that topical ocular hypotensive medication delays or prevents the onset of primary open-angle glaucoma. Arch Ophthalmol. 2002;120:701-713; discussion 829-730.

5. Heijl A, Leske MC, Bengtsson B, Hyman L, Hussein M. Reduction of intraocular pressure and glaucoma progression: results from the Early Manifest Glaucoma Trial. Arch Ophthalmol. 2002;120:1268-1279.

6. Iwase A, Suzuki Y, Araie M, et al. The prevalence of primary open-angle glaucoma in Japanese: the Tajimi Study. Ophthalmology. 2004;111:1641-1648.

7. Zeyen TG, Caprioli J. Progression of disc and field damage in early glaucoma. Arch Ophthalmol. 1993;111:62-65.

8. Harwerth RS, Carter-Dawson L, Smith EL 3rd, et al. Neural losses correlated with visual losses in clinical perimetry. Invest Ophthalmol Vis Sci. 2004;45:3152-3160.

9. Kerrigan-Baumrind LA, Quigley HA, Pease ME, Kerrigan DF, Mitchell RS. Number of ganglion cells in glaucoma eyes compared with threshold visual field tests in the same persons. Invest Ophthalmol Vis Sci. 2000;41:741-748.

10. Quigley HA, Dunkelberger GR, Green WR. Retinal ganglion cell atrophy correlated with automated perimetry in human eyes with glaucoma. Am J Ophthalmol. 1989;107:453-464.

11. Quigley HA, Katz J, Derick RJ, Gilbert D, Sommer A. An evaluation of optic disc and nerve fiber layer examinations in monitoring progression of early glaucoma damage. Ophthalmology. 1992;99:19-28.

12. Sommer A, Katz J, Quigley HA, et al. Clinically detectable nerve fiber atrophy precedes the onset of glaucomatous field loss. Arch Ophthalmol. 1991;109:77-83

13. Mardin CY, Junemann AG. The diagnostic value of optic nerve imaging in early glaucoma. Curr Opin Ophthalmol. 2001;12:100-104.

14. Badala F, Nouri-Mahdavi K, Raoof DA, et al. Optic disk and nerve fiber layer imaging to detect glaucoma. Am J Ophthalmol. 2007;144:724-732.
15. Naithani P, Sihota R, Sony P, et al. Evaluation of optical coherence tomography and heidelberg retinal tomography parameters in detecting early and moderate glaucoma. Invest Ophthalmol Vis Sci. 2007;48: 3138-3145.

16. Kalaboukhova L, Fridhammar V, Lindblom B. Glaucoma follow-up by the Heidelberg retina tomograph - new graphical analysis of optic disc topography changes. Graefes Arch Clin Exp Ophthalmol. 2006;244:654-662.

17. Saarela V, Airaksinen PJ. Heidelberg retina tomograph parameters of the optic disc in eyes with progressive retinal nerve fibre layer defects. Acta Ophthalmol. 2008;86:603-608.

18. Brusini P. Monitoring glaucoma progression. Prog Brain Res. 2008:173:59-73.

19. Nicolela MT, Drance SM. Various glaucomatous optic nerve appearances: clinical correlations. Ophthalmology. 1996;103:640-649.

20. Nicolela MT, McCormick TA, Drance SM, et al. Visual field and optic disc progression in patients with different types of optic disc damage: a longitudinal prospective study. Ophthalmology. 2003;110:2178-2184.

21. Chauhan BC, LeBlanc RP, McCormick TA, Rogers JB. Test-retest variability of topographic measurements with confocal scanning laser tomography in patients with glaucoma and control subjects. Am J Ophthalmol. 1994;118:9-15.

22. Rohrschneider K, Burk RO, Kruse FE, Volcker HE. Reproducibility of the optic nerve head topography with a new laser tomographic scanning device. Ophthalmology. 1994;101:1044-1049.

23. Anderson DR, Patella VM. Automated Static Perimetry (ed 2nd), Mosby, 1999.

24. Zangwill L, Irak I, Berry CC, et al. Effect of cataract and pupil size on image quality with confocal scanning laser ophthalmoscopy. Arch Ophthalmol. 1997;115:983-990.

25. Mikelberg FS, Parfitt CM, Swindale NV, et al. Ability of Heidelberg retinal tomograph to detect early glaucomatous visual field loss. J Glaucoma. 1995;4:242-247.

26. Danesh-Meyer HV, Ku JY, Papchenko TL, et al. Regional correlation of structure and function in glaucoma, using the Disc Damage Likelihood Scale, Heidelberg Retina Tomograph, and visual fields. Ophthalmology. 2006;113:603-611.

27. Bozkurt B, Yilmaz PT, Irkec M. Relationship between Humphrey 30-2 SITA Standard Test, Matrix 30-2 threshold test, and Heidelberg retina tomograph in ocular hypertensive and glaucoma patients. J Glaucoma. 2008; 17:203-210.

28. Ford BA, Artes PH, McCormick TA, et al. Comparison of data analysis tools for detection of glaucoma with the Heidelberg Retina Tomograph. Ophthalmology. 2003;110:1145-1150.

29. Bathija R, Zangwill L, Berry CC, Sample PA, Weinreb RN. Detection of early glaucomatous structural damage with confocal scanning laser tomography. J Glaucoma. 1998;7:121-127.

30. Sawada A, Tomidokoro A, Araie M, Iwase A, Yamamoto T. Refractive errors in an elderly Japanese population: the Tajimi study. Ophthalmology. 2008;115:363-370;e363.

31. Saw SM, Chan YH, Wong WL, et al. Prevalence and risk factors for refractive errors in the Singapore Malay Eye Survey. Ophthalmology. 2008;115:1713-1719.

32. Kempen JH, Mitchell P, Lee KE, et al. The prevalence of refractive errors among adults in the United States, Western Europe, and Australia. Arch Ophthalmol. 2004;122:495-505.

33. Sperduto RD, Seigel D, Roberts J, Rowland M. Prevalence of myopia in the United States. Arch Ophthalmol. 1983;101:405-407.

34. Abe H, Shirakashi M, Tsutsumi T, et al. Laser scanning tomography of optic discs of the normal Japanese population in a population-based setting. Ophthalmology. 2009;116:223-230.

35. Yamazaki Y, Yoshikawa K, Kunimatsu S, et al. Influence of myopic disc shape on the diagnostic precision of the Heidelberg Retina Tomograph. Jpn J Ophthalmol. 1999;43:392-397. 
36. Nakamura H, Maeda T, Suzuki Y, Inoue Y. Scanning laser tomography to evaluate optic discs of normal eyes. Jpn J Ophthalmol. 1999;43:410-414.

37. Park KH, Caprioli J. Development of a novel reference plane for the Heidelberg retina tomograph with optical coherence tomography measurements. J Glaucoma. 2002;11:385-391.

38. Poli A, Strouthidis NG, Ho TA, Garway-Heath DF. Analysis of HRT images: comparison of reference planes. Invest Ophthalmol Vis Sci. 2008;49:3970-3975.
39. Nicolela MT, Walman BE, Buckley AR, Drance SM. Various glaucomatous optic nerve appearances. A color Doppler imaging study of retrobulbar circulation. Ophthalmology. 1996;103:1670-1679.

40. Gardiner SK, Johnson CA, Cioffi GA. Evaluation of the structurefunction relationship in glaucoma. Invest Ophthalmol Vis Sci. 2005;46:3712-3717.

\section{Publish your work in this journal}

Clinical Ophthalmology is an international, peer-reviewed journal covering all subspecialties within ophthalmology. Key topics include: Optometry; Visual science; Pharmacology and drug therapy in eye diseases; Basic Sciences; Primary and Secondary eye care; Patient Safety and Quality of Care Improvements. This journal is indexed on

\footnotetext{
Submit your manuscript here: http://www.dovepress.com/clinical-ophthalmology-journal
}

\section{Dovepress}

PubMed Central and CAS, and is the official journal of The Society of Clinical Ophthalmology (SCO). The manuscript management system is completely online and includes a very quick and fair peer-review system, which is all easy to use. Visit http://www.dovepress.com/ testimonials.php to read real quotes from published authors. 